Musées, Patrimoine et Culture scientifiques et techniques

$194 \mid 2021$

mars-avril 2021

\title{
Variations des imaginaires. De l'amour au Palais de la découverte
}

Astrid Aron et Maud Gouy

\section{OpenEdition}

Journals

Édition électronique

URL : https://journals.openedition.org/ocim/4228

DOI : $10.4000 /$ ocim. 4228

ISSN : 2108-646X

Éditeur

OCIM

Édition imprimée

Date de publication : 1 mars 2021

Pagination : 10-17

ISSN : 0994-1908

Référence électronique

Astrid Aron et Maud Gouy, « Variations des imaginaires. De l'amour au Palais de la découverte », La

Lettre de l'OCIM [En ligne], 194 | 2021, mis en ligne le 01 mars 2021, consulté le 12 avril 2022. URL: http://journals.openedition.org/ocim/4228 ; DOI : https://doi.org/10.4000/ocim.4228

Ce document a été généré automatiquement le 12 avril 2022.

Tous droits réservés 


\title{
Variations des imaginaires. De l'amour au Palais de la découverte
}

\author{
Astrid Aron et Maud Gouy
}

Création de l'illustratrice Anna Wanda Gogusey pour l'exposition De l'amour (détail).

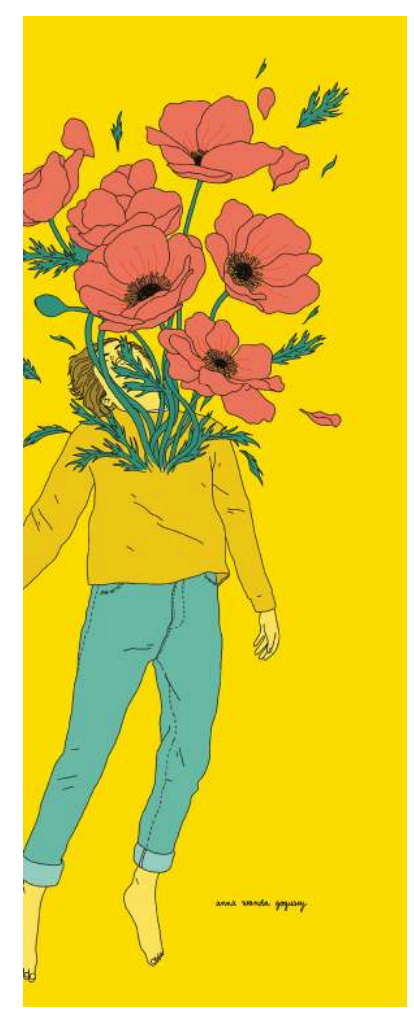

(C) A. W. Gogusey/EPPDCSI

L'exposition De l'amour a ouvert du 8 octobre 2019 au 27 septembre 2020 au Palais de la découverte à Paris; elle est installée depuis le 10 décembre 2020 au Quai des savoirs à Toulouse, en attente de recevoir son public pour cause de pandémie et avant de partir 
vers d'autres lieux d'accueil. Que viennent chercher les visiteurs à travers ce type de problématique ? Comment et pourquoi un sujet comme "l'amour » trouve-t-il sa place dans un centre de sciences? Moteur tout-puissant de tant d'histoires, tristes ou joyeuses, il reste difficile à cerner. Est-il universel? Singulier? Exposer l'amour, le désir, la sexualité, le genre dans un musée de sciences nous a interrogé sur ce qu'est l'amour en 2020.

2 Affirmer que la sexualité est une question politique est une évidence en 2020. Les politiques publiques et les associations féministes, gays, LGBTQIA+ ont participé et participent à faire passer ce que l'on croyait intime de la zone privée à la zone publique. Philosophes et sociologues s'accordent là-dessus. L'amour comme on l'entend aujourd'hui est, lui, récent puisqu'il date du XIX ${ }^{e}$ siècle. À la fin du XIX ${ }^{e}$ siècle, l'exode rural et l'industrialisation modifient le système matrimonial : l'implication des parents s'amoindrit, les enjeux familiaux laissent place à un nouveau modèle du mariage qui donne naissance aux codes de l'amour romantique. Le sentiment amoureux devient l'origine et non la conséquence de l'union. Il prend alors une importance sociétale nouvelle et devient un enjeu majeur.

3 Nous présentons dans cet article les partis-pris choisis, les articulations entre art et science, intime et sociétal, désir et construction. Nous proposons une lecture critique de la méthodologie d'une exposition, dont la thématique universelle touche chacun, chacune.

\section{En quatre mots : l'amour}

Comment rendre compte de ce thème dans un centre de sciences, le Palais de la découverte? Ce sujet vient se glisser dans les interstices des sphères sociales et politiques, personnelles et universelles, culturelles et scientifiques. La clé d'entrée a été l'utilisation de quatre mots grecs :

- éros, le désir, la passion charnelle ;

- storgê, l'amour familial ;

- agapè, l'amour désintéressé ;

- philia, l'amitié, le lien social.

La langue grecque est fertile et ces quatre termes permettent de cerner les contours du territoire de l'amour. Issue de «la maison des mots» comme l'écrit Andrea Marcolongo dans $L a$ langue géniale ${ }^{1}$, la langue grecque recèle des manières de s'exprimer, de formuler des mots et des idées qui ne trouvent pas d'expression exacte dans notre langue. En effet, là où le français n'a qu'un terme pour désigner l'amour pour un ami ou pour le chocolat, la langue grecque permet les nuances. 
Un système graphique dans la galerie des attachements pour indiquer les formes d'amour évoquées dans les installations.

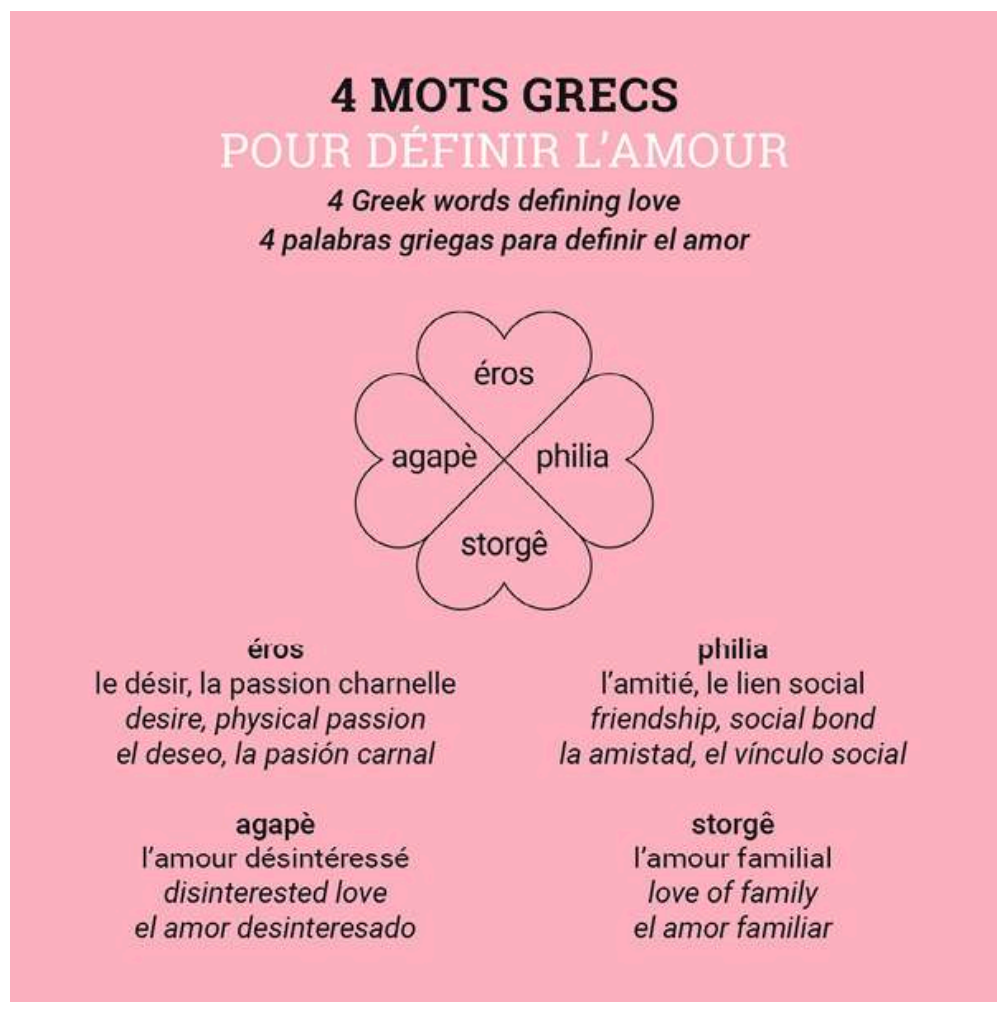

\section{(C) EPPDCSI}

Examiner l'amour à l'aune de ses quatre traductions grecques campe l'étendue du sujet et le cerne. Il aurait pu être plus vaste encore et s'étendre aux attachements qui nous lient aux lieux, aux objets, aux animaux, aux plantes. Mais exposer c'est aussi choisir. Et nous avons fait le choix, dans l'exposition, de n'explorer l'attachement qu'entre êtres humains. Les visiteurs se retrouvent ainsi équipés, avec les quatre mots grecs, d'une grille de lecture qui affirme l'étendue de l'exploration proposée : il s'agit d'embrasser tout à la fois le sentiment amoureux, l'amour familial, l'amitié et l'amour du genre humain. Et loin de compartimenter chacune de ces formes d'amour, nous les avons volontairement mélangées, au sein de la galerie des attachements, dans un joyeux bricà-brac.

11 Car il est non seulement très fastidieux de les séparer mais impossible. C'est ce que nous révèlent les biologistes dans la deuxième galerie de l'exposition. Les supports biologiques de l'amour, les hormones, les neurotransmetteurs, ne sont pas dédiés l'un à éros, l'autre philia ou storgé. Ils participent, en proportion variable, à chacun de ces sentiments. Même imbrication dans le cerveau. Les zones du cerveau impliquées ne sont pas spécifiques à une forme d'amour ni même au seul sentiment d'attachement. Aux dires du philosophe Francis Wolff ${ }^{2}$, la relation amoureuse peut même s'interpréter comme une composition d'au moins deux des trois ingrédients que sont la passion, l'amitié et le désir. L'exploration de la galerie des attachements se fait donc épaulée par un système graphique qui labellise chaque contenu à l'aide de plusieurs mots grecs car il n'y a pas de frontières imperméables entre les différentes formes d'amour. 


\section{La galerie des attachements}

La peinture, la sculpture, les romans d'amour, les gravures ont été, en Occident, des modèles dans la construction amoureuse, et ce, dès le Moyen Age, puis au XIX ${ }^{e}$ siècle et au début du $\mathrm{XX}^{\mathrm{e}}$. En témoignent la condamnation de Baudelaire à une forte amende et la censure de six pièces jugées immorales des Fleurs du mal, ou encore le blâme adressé à Gustave Flaubert pour le roman Madame Bovary qualifié par le procureur Ernest Pinard d'un "réalisme vulgaire et souvent choquant de la peinture des caractères "3. Hier Foucault, Pasolini, Moravia, de Beauvoir, Genet... aujourd'hui Preciado, Despentes... mais aussi Antigone, punk à sa façon modèlent nos imaginaires. Ces textes viennent interroger la norme et permettent à chacun, chacune de créer ses propres repères. Le cinéma et les sites porno jouent également un rôle majeur dans la construction de la sexualité et de nos relations affectives et propulsent l'amour et la sexualité de la sphère intime dans le politique et le social.

La galerie des attachements, bric-à-brac de références culturelles.

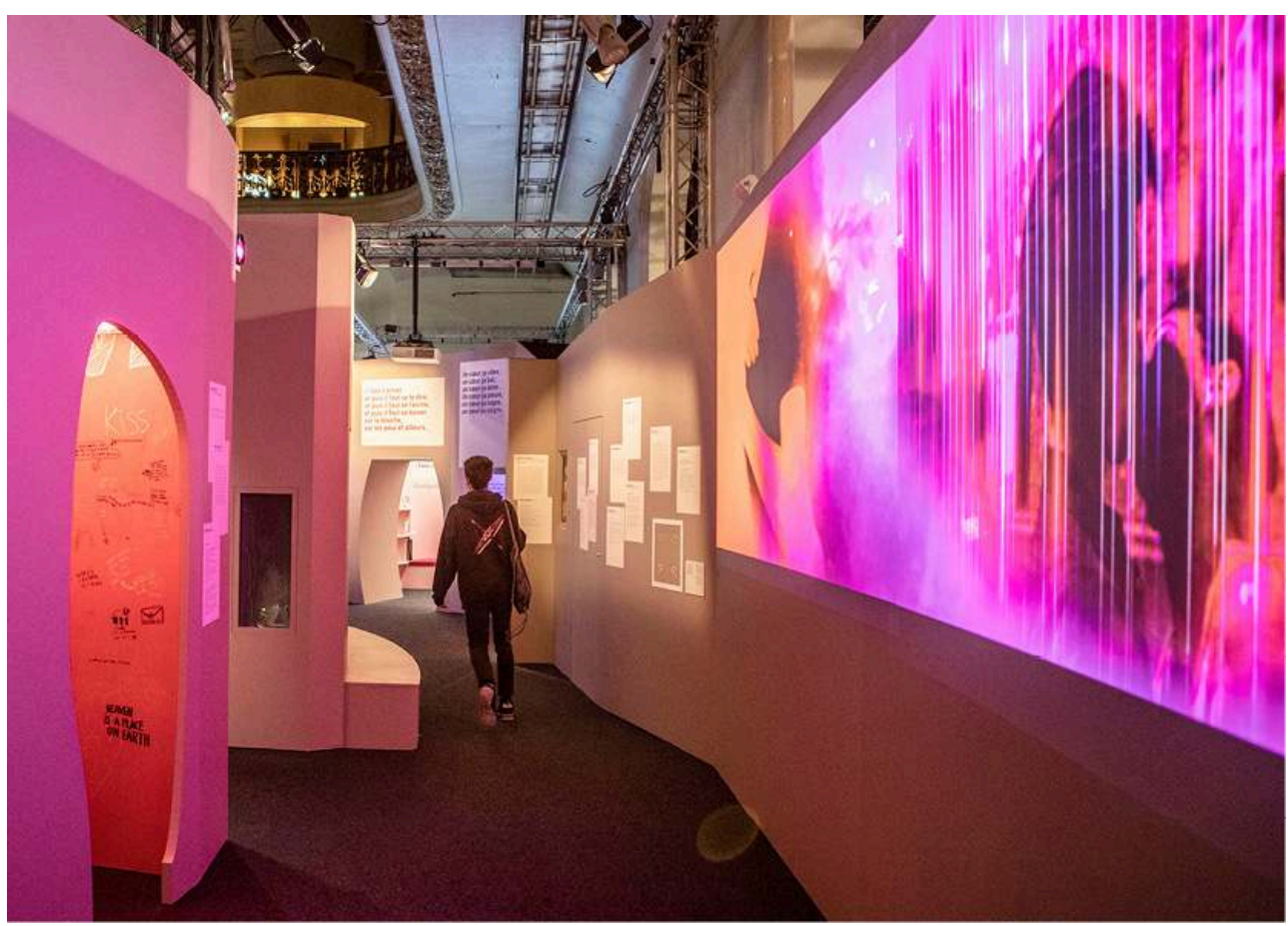

(C) N. Breton/EPPDCSI

Cette galerie propose une vingtaine de saynètes éclectiques. De Stendhal à Marivaux en passant par le téléphone portable et l'amour de soi... autant de références culturelles et populaires, nullement exhaustives tant le palmarès est immense. Se côtoient Le petit poucet, Le petit Nicolas, un film érotique, la madeleine de Proust, des textes de Duras, d'Éluard, de jeunes auteurs Julie et Éléonor Gilbert, Maria Pia Duponchelle ou encore un texte de Primo Levi sur le déracinement absolu sans oublier une vaste bibliothèque et des contes de Chine, d'Ethiopie, du Vietnam, de Grèce ou encore une légende sioux - à écouter dans une alcôve.

Une montagne de doudous, pour témoigner des premiers attachements vont ricocher sur des reproductions de tableaux de Watteau, de Pierre et Gilles ou de Man Ray. Ces 
contrepoints ont tous un même statut et se côtoient formant un bric-à-brac orchestré par le scénographe Raphaël Lerays, qui sollicite la mémoire et la sensibilité du visiteur.

\section{Parti pris : Mettre en scène un paradoxe qui est de lutter contre l'injonction à être en couple tout en mettant en scène l'amour en ligne}

Les sociologues ont une bonne longueur d'avance pour expliquer les liens amicaux, familiaux et conjugaux. Aujourd'hui leur recherche s'étend également aux rencontres sur les réseaux sociaux. La lecture du livre Happycratie d'Edgar Cabanas et Eva Illouz ${ }^{4}$ a confirmé notre choix de battre en brèche l'idée, "sournoise", de l'injonction au bonheur. Le bonheur comme une quête, une recherche ultime, qui passe nécessairement par le couple avec un grand $\mathrm{C}$ où chacun doit être heureux tous les jours. Nous ne souhaitions pas, au sein de cette exposition, ériger le couple comme modèle, d'où le souhait de faire flirter les quatre facettes de l'amour sur un pied d'égalité. De plus, Marie Bergström, chercheuse à l'Institut national d'études démographiques (Ined) nous a montré, enquêtes à l'appui, que les pratiques numériques n'ont pas bouleversé la géographie amoureuse en France sauf pour les personnes homosexuelles qui utilisent principalement ce moyen pour trouver leurs partenaires. Les sites et applications de rencontres ont, a priori, la capacité de mettre en contact des individus éloignés spatialement et socialement. Pourtant, la tendance à former des couples au sein du même milieu social reste la même qu'avec les autres modes de rencontres: de nouveaux mécanismes de sélection de la part des partenaires sont à l'œuvre. Sans être une révolution, le recours aux outils numériques modifie à la marge certains aspects de la rencontre : importance des échanges textuels, importance de l'image, rencontres à l'abri des regards. Dans ce cadre, l'écriture a, en effet, une grande importance.

Morgane Ortin autrice, l'a bien compris. Elle accorde une légitimité aux écrits contemporains échangés pas SMS et grâce aux médias sociaux. Son travail d'édition sur le compte Instagram @amours_solitaires donne un statut épistolaire à des échanges qui relèvent de l'ordinaire. Dans l'exposition, les visiteurs consultent cette nouvelle prose, soigneusement sélectionnée par Morgane Ortin et relevant de l'amour familial, l'amour fraternel, l'amitié, les déclarations d'amour, les sextos, la rupture, la séduction et du chagrin d'amour. 
Grandes illustrations jalonnant le parcours et montrant une diversité de situations de genre.

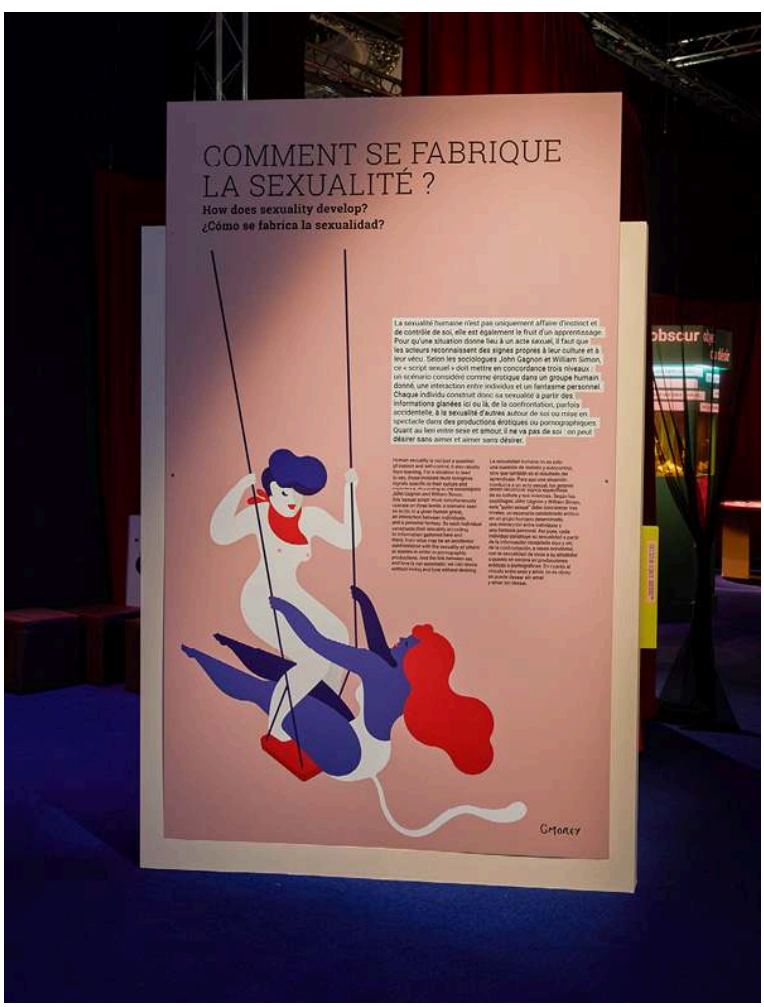

(c) EPPDCSI

\section{Parti pris : Exposer l'amour en tenant compte de la question du genre}

Sensibilisés à la question depuis quelques années, la chasse aux stéréotypes de genre est devenue un exercice familier des centres de science. L'écriture inclusive est venue s'ajouter à la boîte à outils qui comprenait déjà le langage épicène ${ }^{5}$.

Mais dans une exposition sur l'amour, cette exigence prenait une dimension particulière : il fallait déjouer les rôles assignés aux genres dans la relation amoureuse et prendre en compte tous les types d'orientations sexuelles, à la fois dans les représentations visuelles et dans l'écriture des textes. À chaque média sa solution. Concernant les représentations visuelles, les illustrations des panneaux ou les dessins réalisés pour les animations, nous avons veillé à présenter une diversité de situations de genres, d'orientations mais aussi de physiques et d'âges. Nous avons également eu recours à des représentations symboliques, qui pouvaient tout aussi bien figurer un personnage appartenant à un sexe qu'à un autre.

Comment parler d'amour sans poser la question de l'orientation du désir? Qu'est-ce qui fait que certains individus nous attirent et d'autres pas du tout? Traiter de l'orientation sexuelle, suppose d'éclairer la notion de genre... et de prendre le temps nécessaire aux subtilités. La forme audiovisuelle et le discours s'imposaient. La réalisation d'un film, dédié à cette question, a été confiée aux Chevreaux suprématistes. Ils ont habilement mis au point une grammaire visuelle, basée sur des mains filmées qui déplacent des images symboliques. Ces mains figurent tour à tour des personnages, ni 
hommes ni femmes. Elles imitent l'anatomie des organes sexuels. Ce détour par l'objet symbolique et la main, agrémenté du ton humoristique du film, a permis de traiter habilement des questions de sexe, de genre et d'orientation sexuelle. Concernant la rédaction des textes, la contrainte s'est avérée finalement légère. Il a suffi de centrer le discours, non sur les protagonistes, mais sur la relation elle-même. Cette facilité à gommer les étiquettes est révélatrice. L'amour se définit aisément, dans sa singularité autant que dans son universalité, en dehors des catégories.

Enfin, une parité stricte a été appliquée dans le choix des auteurs de citations et de poèmes qui figuraient dans la galerie des attachements: autant d'hommes que de femmes.

\section{Présenter et représenter : trois défis}

Comment présenter et représenter la sexualité, l'érotisme dans un musée de sciences, comment faire émerger le corps? Le corps à corps? La sensualité, les fantasmes, la violence sexuelle, sans choquer les visiteurs? Des objets, des images, des photos, des sculptures, des statuettes... ce qui est obscène pour une personne ne l'est pas nécessairement pour une autre. Où se situe la frontière? On voit bien que ce sujet vient se glisser dans les interstices de l'intime. Toutes ces sensations liées au corps, banales et universelles, sont complexes à mettre en scène au Palais de la découverte. Nous avons opté pour un film de 7 minutes. Dans la commande, nous avons bien précisé que nous ne souhaitions pas de rapport sexuel stéréotypé, pas de rôles figés. Manon Heugel, la réalisatrice a bien pris la mesure du cahier des charges et a proposé, à travers une danse, une mise en scène du désir, du fantasme et de la jouissance ; en filigrane, ce film vient détourner les codes de la pornographie et déconstruire le mythe de l'homme viril et d'une femme soumise par une inversion des codes, des corps... "Cette chorégraphie montre un rapport sexuel sous forme stylisée. On y voit un homme noir et une femme blanche se déshabiller, échanger leurs sous-vêtements, danser, rouler et s'étreindre. Pas de rôles figés, ni de mâle invulnérable, ni de femme réduite à regarder le plafond "».

\section{Centrer le discours sur la relation permet d'éviter les stéréotypes de genre \\ Extrait d'un texte accompagnant un jeu sur les preuves d'amour : \\ «Donne-moi des preuves.}

La relation amoureuse se traduit par des échanges, des dons et des contre-dons. On donne de son temps, on partage son cercle d'amis, sa famille, on fait don de son corps. Le processus de mise en couple répond également à des codes. Par exemple, l'un des actes constitutifs de la formation du couple est de s'offrir des cadeaux ou prendre des vacances ensemble. L'absence de l'une de ces pratiques au sein d'une relation amoureuse peut être source de reproches et de souffrance ».

Deux autres défis se sont imposés à notre réflexion muséographique. Pour mémoire, le travail de muséographe consiste à inventer un dispositif, un artefact qui est porteur d'un concept ou d'une pensée. En l'occurrence ici, comment inventer un «truc » qui mette en scène l'idée que la sexualité humaine n'est pas uniquement une affaire 
d'instinct et de contrôle de soi, et qu'elle est également le fruit d'un apprentissage. Chaque individu construit donc sa sexualité à partir des informations glanées ici ou là, de la confrontation, parfois accidentelle, à la sexualité d'autres autour de soi ou mise en spectacle dans des productions érotiques ou pornographiques. La sexualité est fondamentalement apprise. Elle se construit donc sur un paradoxe: la sexualité humaine se vit en cachette, en aparté, tout en nécessitant un apprentissage. Forts de ces données, nous avons imaginé un jeu qui puisse mettre en forme cette complexité sociologique et sémantique. Comment faire se corréler les mots comme "masturbation», «découverte du coït», «porno» mais aussi "attouchement», « inceste » sans heurter la sensibilité et/ou la mémoire du visiteur et, de fait, lui fermer la porte de cet élément? Avec Mitia Notaras et Géraldine Grammon, les designers de l'exposition, nous avons imaginé un jeu de construction dont les pièces légendées sont à faire tenir en équilibre, une sorte de mobile qui invite le visiteur à agencer des mots et expressions qui peuvent témoigner de la construction de sa sexualité. Les pièces du jeu portent chacune une image évocatrice ou un texte comme "une planche d'anatomie suggestive, un poème érotique, des films pornos, des romans photos, un slow, un baiser volé, etc.». Ce jeu offre aussi la possibilité aux visiteurs qui ne souhaitent pas reconstruire littéralement le cheminement de leur sexualité, de réfléchir, par la simple lecture des mots, aux types d'influences que celle-ci a pu recevoir.

Image extraite du film Eros de Manon Heugel de Bridges.

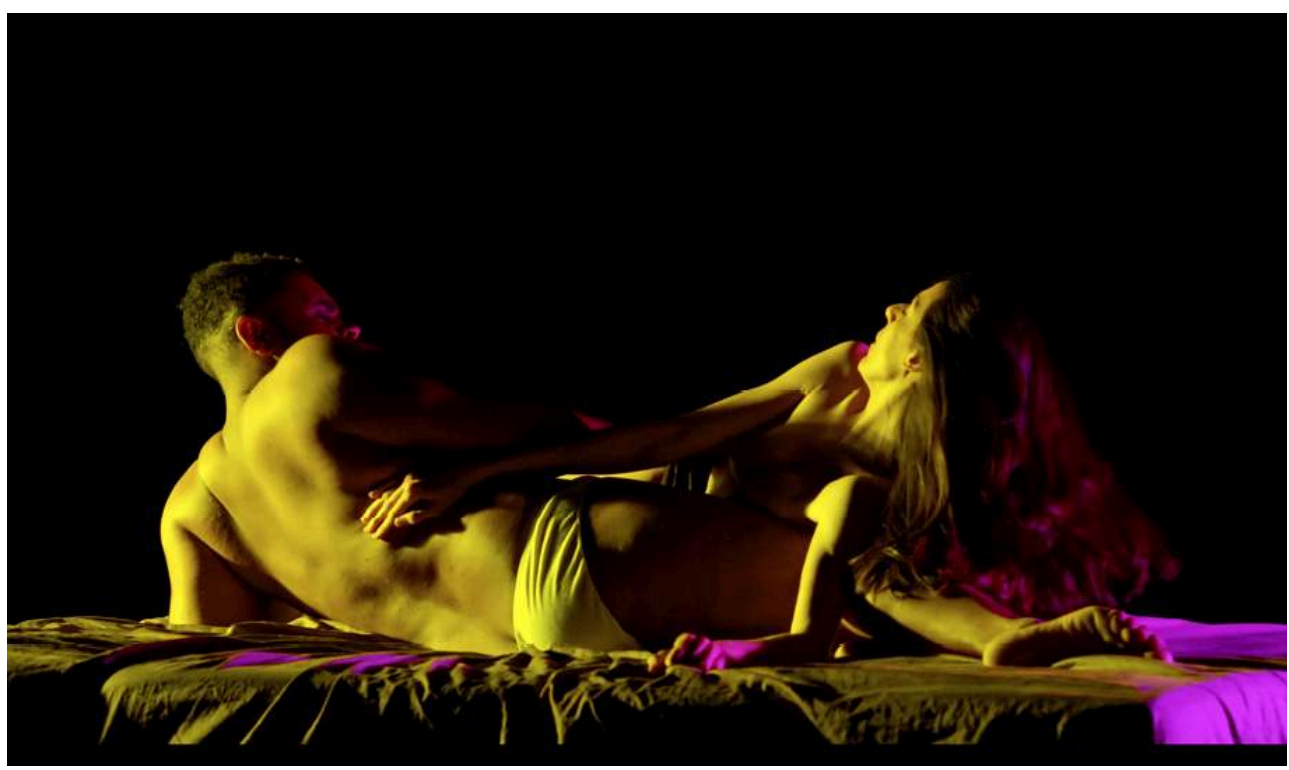

(C) M. Heugel de Bridges/EPPDCSI

Troisième défi, comment arriver à mettre en scène le désir sans le rendre stéréotypé. L'objet érotique universel n'existe pas. Un objet peut être chargé d'érotisme pour certains et en être totalement dénué pour d'autres. En réalité, nos désirs sont intimement liés aux constructions sociales et aux stéréotypes qui nous entourent. Ils sont le fruit d'un imaginaire qui varie selon les périodes de l'histoire, les cultures, les pays. Nous ne souhaitions pas proposer des objets types comme un talon aiguille ou des bas qui, même s'ils sont objets de fantasmes, n'évoquent pas le rapport intime que chacun caresse par son imaginaire, sa sensibilité son contexte... C'est donc une installation sonore en écho à des objets disparates appartenant à des vécus particuliers 
et des cultures diverses qui relate cette objectivation du désir dans sa diversité identitaire, sexuelle et sensorielle.

Jeu de construction invitant à se remémorer les éléments qui ont participé à la construction de sa propre sexualité.

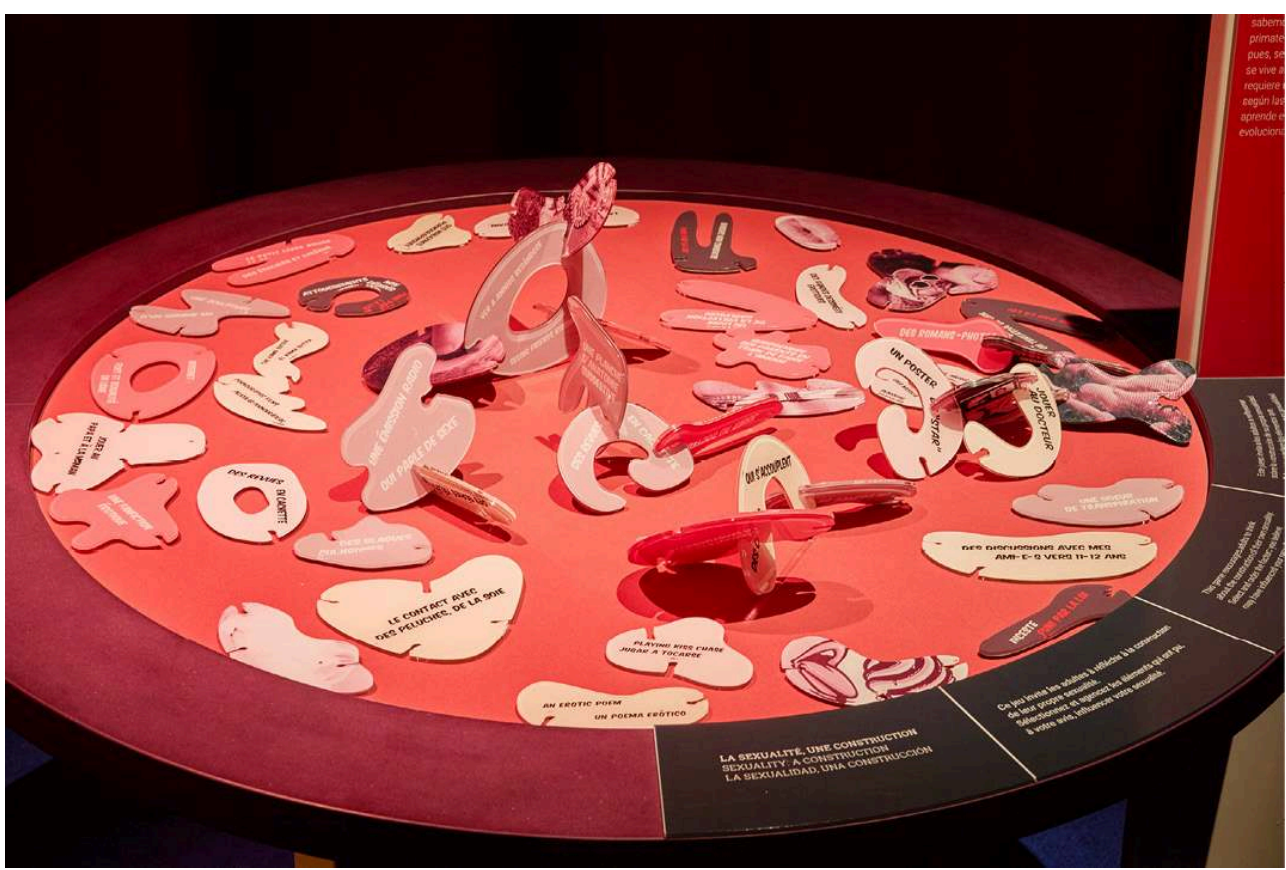

(c) EPPDCSI

\section{Les échos entre les deux galeries (arts et sciences)}

De l'amour aurait pu être nommé Fragments d'un discours scientifique, clin d'œil et hommage à Roland Barthes pour son magnifique ouvrage Fragments d'un discours amoureux $x^{7}$ paru il y a 43 ans, qui assemble et tresse des œuvres poétiques, théâtrales, mais aussi des tableaux et de la musique dans le but de cerner le territoire de l'amour. Mais c'est aussi l'idée que ce sujet peut être raconté par des artistes et exploré par des scientifiques. L'idée que les disciplines, là encore, interagissent, voire se consolident, se contredisent en tentant de définir cette sphère complexe. Il est aisé de faire le lien entre la cristallisation stendhalienne et les neurosciences qui, images à l'appui, peuvent confirmer que le concept de la cristallisation inventé par Stendhal est un phénomène d'idéalisation qui naît au début d'une relation amoureuse. L'amoureux a tendance à attribuer à l'être aimé des qualités qui sont en réalité le fruit de ses fantasmes. Dans son livre De l'amour, publié en 1822, Stendhal exprime sa passion: "Ce que j'appelle cristallisation, c'est l'opération de l'esprit, qui tire de tout ce qui se présente la découverte que l'objet aimé a de nouvelles perfections ». Et justement, les neuroscientifiques observent lors de la rencontre amoureuse et du désir naissant, que tout le cerveau loin d'être en ébullition voit certaines zones cérébrales se désactiver. Selon leurs études, c'est le cas de l'amygdale, une structure cérébrale qui joue un rôle important dans la peur. Cela nous aiderait-il à nous rapprocher de l'autre? Le cortex préfrontal, impliqué dans le jugement critique, est lui aussi désactivé. Est-ce pour ça que l'on discerne moins les défauts de la personne qu'on aime? L'amour rend-il aveugle ou favorise-t-il la 
cristallisation au sens stendhalien? Ainsi, la cristallisation stendhalienne, évoquée dans la première partie de l'exposition, se voit éclairée, dans la suite du parcours de visite, par les recherches récentes utilisant l'imagerie cérébrale.

Cette exposition, présentée dans un contexte difficile (Covid-19) a, malgré la fermeture de l'établissement et des mesures drastiques d'ouverture après le confinement, accueilli 144000 visiteurs. Nous avons observé sans pouvoir l'évaluer une proportion de jeunes gens supérieure à la moyenne habituelle. Les verbatim du livre d'or laissent apparaître des déclarations d'amour éclectiques, adressées aux êtres aimés mais également à l'exposition et au genre humain.

\section{NOTES}

1. Marcolongo A. La Langue géniale - 9 bonnes raisons d'aimer le grec. Traduit par Béatrice RobertBoissier. Paris : Les Belles Lettres, 2018, 202 p.

2. Wolff F. Il n'y a pas d'amour parfait. Paris : Fayard, 2016, 72 p.

3. Genin C. À propos de l'œuvre, Flaubert, Madame Bovary, Les essentiels Littérature, BnF Gallica : https://gallica.bnf.fr/essentiels/flaubert/madame-bovary/propos-oeuvre

4. Cabanas E. et Illouz E. Happycratie : Comment l'industrie du bonheur a pris le contrôle de nos vies. Paris : Premier Parallèle, 2018, 260 p.

5. Il s'agit d'avoir recours aux formes textuelles dont le genre grammatical est aussi bien féminin que masculin comme c'est le cas pour les mots " personne ", « individu », " autrui », « sujet ». Il est à noter que la langue anglaise devient totalement épicène par le passage au pluriel.

6. Mazaurette M. Les hommes au défi du rapport sexuel idéal, Le Monde.fr, 13 octobre 2019 : https://www.lemonde.fr/m-le-mag/article/2019/10/13/les-hommes-au-defi-du-rapport-sexuelideal_6015298_4500055.html

7. Barthes R. Fragments d'un discours amoureux. Paris : Seuil, 1977, 288 p.

\section{RÉSUMÉS}

Un cheminement à travers l'exposition De l'amour est proposé par les deux commissaires de cette exposition. Elles interrogent le sens du parcours, leurs partis-pris et explicitent les choix muséographiques opérés pour approcher ce sujet dans un centre de sciences, à la croisée des sphères sociales et politiques, personnelles et universelles, culturelles et scientifiques. 
INDEX

Mots-clés : exposition, muséographie, centre de sciences, CCSTI, musée scientifique, technique et industriel

\section{AUTEURS}

\section{ASTRID ARON}

Muséographe à Universcience et commissaire de l'exposition De l'amour. astrid.aron@universcience.fr

\section{MAUD GOUY}

Muséographe à Universcience et commissaire de l'exposition De l'amour. maud.gouy@universcience.fr 